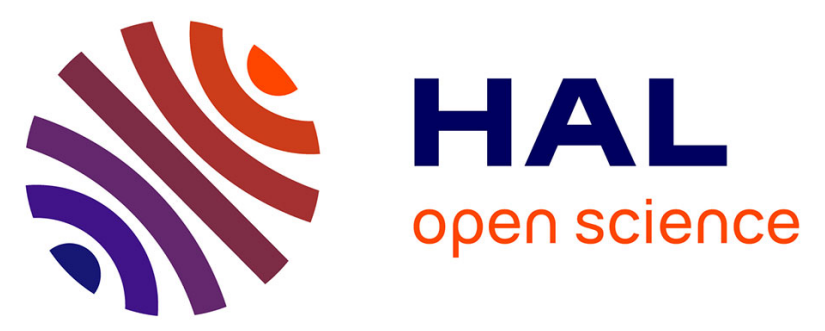

\title{
Prediction performance of reservoir computing systems based on diode-pumped Erbium doped microchip laser subject to optical delayed feedback
}

Romain Modeste Nguimdo, Eric Lacot, Olivier Jacquin, Olivier Hugon, Guy van Der Sande, Hugues Guillet

\section{To cite this version:}

Romain Modeste Nguimdo, Eric Lacot, Olivier Jacquin, Olivier Hugon, Guy van Der Sande, et al.. Prediction performance of reservoir computing systems based on diode-pumped Erbium doped microchip laser subject to optical delayed feedback. Optics Letters, 2017, 10.1364/OL.42.000375 . hal01450190

\author{
HAL Id: hal-01450190 \\ https://hal.science/hal-01450190
}

Submitted on 31 Jan 2017

HAL is a multi-disciplinary open access archive for the deposit and dissemination of scientific research documents, whether they are published or not. The documents may come from teaching and research institutions in France or abroad, or from public or private research centers.
L'archive ouverte pluridisciplinaire HAL, est destinée au dépôt et à la diffusion de documents scientifiques de niveau recherche, publiés ou non, émanant des établissements d'enseignement et de recherche français ou étrangers, des laboratoires publics ou privés. 


\title{
Prediction performance of reservoir computing systems based on diode-pumped Erbium doped microchip laser subject to optical delayed feedback
}

\author{
Romain Modeste Nguimdo ${ }^{1, *}$, Eric Lacot $^{2}$, Olivier Jacquin ${ }^{2}$, Olivier Hugon ${ }^{2}$, Guy Van der \\ SANDE $^{3}$, AND Hugues Guillet de Chatellus ${ }^{2}$ \\ ${ }^{1}$ Optique Nonlinéaire Théorique, Université Libre de Bruxelles, Campus Plaine, CP 231, 1050 Bruxelles, Belgium \\ 2 Université Joseph Fourier, Grenoble Alpes, CNRS, LIPhy, F-38000 Grenoble France \\ ${ }^{3}$ Applied Physics Research Group, Vrije Universiteit Brussel (VUB), 1050 Brussels Belgium \\ *Corresponding author: Romain.Nguimdo@vub.ac.be \\ Compiled December 18, 2016
}

Reservoir computing (RC) systems are computational tools for information processing which can be fully
implemented in optics. Here, we experimentally and numerically show that an optically pumped laser
subject to optical delayed feedback can yield similar results as those obtained for electrically pumped
lasers. Unlike the previous implementations, the input data is injected at a time interval which is much
larger than the time-delay feedback. This data is directly coupled to the feedback light beam. Our results
illustrate new possible avenues for RC implementations for prediction tasks. $\odot 2016$ Optical Society of America

OCIS codes: (0000.0000) Lasers.

http://dx.doi.org/10.1364/ao.XX.XXXXXX

Reservoir computing (RC) is a brain-inspired concept for information processing which has been recently demonstrated to be efficient for solving practical time dependent tasks [1, 2]. $\mathrm{RC}$ systems operate by ensuring a nonlinear mapping between the input and the output allowing therefore a variety of information processing through training. To perform well, an RC system typically requires high dimensionality and nonlinearity. Traditionally, a high dimensionality is obtained by randomly interconnecting a large number of neurons while the needed nonlinearity can be implemented through sigmoidal activation functions. For example, with an ensemble of 16 interconnected semiconductor optical amplifiers, state-of-the-art performance has been achieved [3].

Alternatively, state-of-the-art performance also has been obtained by relying on a single dynamical nonlinear node subject to delayed-feedback [4]. This configuration (typically referred to as the delay-based RC) has the advantage of being easy to train and to implement experimentally. It has led to several implementations even at high processing speeds using stand-alone commercial telecommunication components [5-8]. The main differences between these experiments are the type of nonlinearity used and how the input matches with the period of the delay line. In those implementations, the nonlinear response of the reservoir is provided by passive nonlinearity such as saturable absorption of a semiconductor mirror [9-11] or by active devices such as optoelectronic modulators [5, 8], optical amplifiers
[3] or semiconductor lasers [7]. These experiments have been supported by numerical simulations [8, 12-15]. Numerical simulations also have shown that the different modes of multi mode lasers subject to optical delayed feedback can be independently used to process in parallel independent tasks [16]. In all cases, the readout layer is trained (using some form of regression) from the state vectors of the reservoir in response to the training data.

Until now, only one experiment has been dedicated to RC systems in which the processing is done from the response provided by a laser subject optical delayed feedback [7]. The laser used in this experiment was an electrically pumped single longitudinal mode laser and the input data was either electrically injected by modulating the pump current or optically injected into the reservoir through optical injection using another laser. Note that in others experiments [5, 6, 8-11], lasers are used as light source to supply the reservoir.

In this work, we pursue three different objectives: (i) we aim at investigating whether a different type of laser subject to similar delayed feedback can yield similar results as those obtained in [7]. More precisely, we experimentally and numerically investigate whether an optically pumped laser can produce similar results as electrically pumped lasers. (ii) In the usual procedure of $\mathrm{RC}$, the injection times of the input data (i.e., the inverse of the processing speeds) is close or corresponds to the time delay. In addition in previous experiments, long time-delays (time-delays much larger than the system's characteristic time) have been 


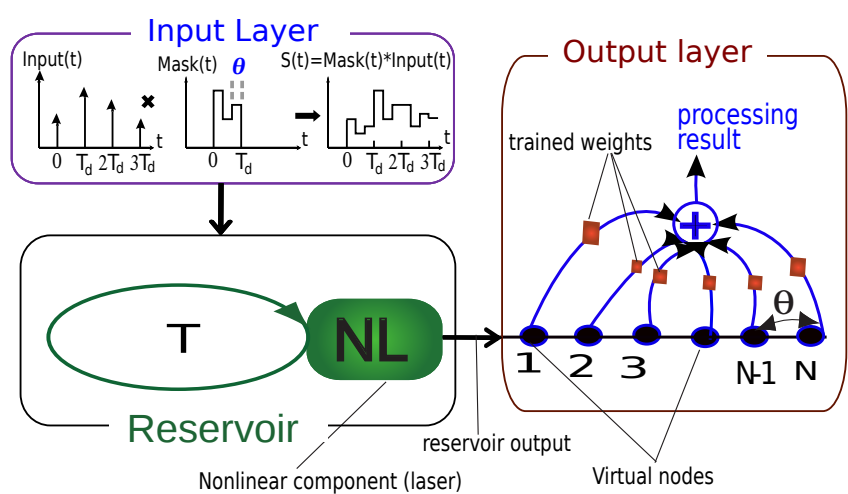

Fig. 1. Conceptual scheme of RC based on a nonlinear node (laser) with delayed feedback.

used $[4-14,16]$. We explore here whether time-delays less or comparable to laser relaxation time can also be suitable for RC systems. This approach is interesting since short delay lines are desirable for on-chip implementations because it consumes less wafer space. The time-delays will be typically fixed during the manufacturing phase for real-world systems while the processing speeds can be controlled. We also investigate the effect of such a change for fixed time-delay. (iii) we also want to explore whether the data can be directly coupled to the feedback light beam instead of using an additional laser for optically injecting the electrical data into the reservoir as done in [7].

RC system can be conceptually divided into three blocks, which are schematically shown in Fig. 1: an input layer, a reservoir and an output layer. The input layer is the stage where the input data is provided and preprocessed before being injected into the reservoir for their processing. The preprocessing includes the rescaling of the input signal and its multiplication by a mask matrix $M$ that defines the coupling weights from input to the reservoir. The role of the mask is to ensure the variability of the signal over the different virtual nodes where the information is read out. These virtual nodes are formed by sampling the delay line at a fixed time interval $\theta$. The output layer is the stage where the different node responses are weighted and linearly summed up. The optimal values of these weights are those for which the summation of all the different node responses always approaches the associated target as closely as possible. They can be typically determined with an off-line training procedure using digital computers [5-8, 11-14] or an on-line training procedure using an FPGA (Field-Programmable Gate Array) [17]. In our case, the training is done off-line. Three time scales are relevant: the data injection time $T_{d}$, the feedback time-delay $T$ and the mask length given by $N \theta$ where $N$ is the number of virtual (temporal) nodes. We consider $T_{d}=N \theta$ and apply this same duration during the postprocessing of each data point.

As benchmark task, we will use the Santa time series to evaluate the prediction performance of the system. The Santa-Fe data are intensity time series experimentally recorded from a far-infrared laser operating in a chaotic state [18]. The goal for this task is to predict the sample one-step ahead in a chaotic time trace before it has been injected into the reservoir computer. The system performance is evaluated by calculating the normalized mean square error (NMSE) between the predicted value $y$ and

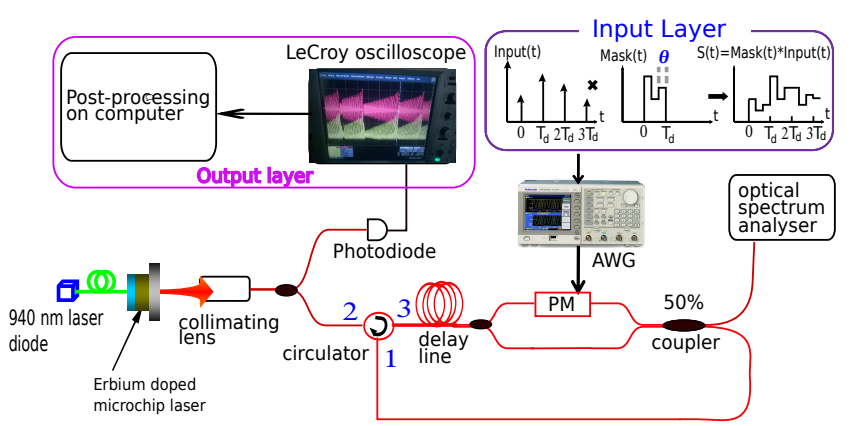

Fig. 2. Experimental setup: PM: phase modulator; AWG: Arbitrary waveform generator.

the expected value $\bar{y}$ :

$$
\operatorname{NMSE}(\mathbf{y}, \overline{\mathbf{y}})=\frac{\left\langle\|\mathbf{y}(\mathbf{n})-\overline{\mathbf{y}}(\mathbf{n})\|^{2}\right\rangle}{\left\langle\|\overline{\mathbf{y}}(\mathbf{n})-\langle\overline{\mathbf{y}}(\mathbf{n})\rangle\|^{2}\right\rangle}
$$

where $n$ is a discrete time index while $\|$.$\| and \langle$.$\rangle stand for$ the norm and the average over time, respectively. Note that $\mathrm{NMSE}=0$ means perfect prediction while NMSE $=1$ indicates no prediction at all. Our results will be compared to those in [7] as it is a similar RC system which was, however, built using a different type of laser and a large time-delay.

The Experimental setup is shown in Fig. 2. It is composed of a diode-pumped Erbium doped microchip laser with optical delayed feedback. The microchip laser is a sample provided by the laboratory LETI-CEA (Technologies Avanckes) [19]. The threshold currents of the diode-pump and the micro laser are $I_{s p} \approx 24.2 \mathrm{~mA}$ and $I_{s} \approx 100 \mathrm{~mA}$, respectively. The microchip laser's stability threshold is close but above its lasing threshold. The micro laser is bi-mode which, above $I_{s}$, emits a total radiation of a few milliwatts at the wavelength $\lambda_{1} \sim 1532 \mathrm{~nm}$ and $\lambda_{2} \sim 1535 \mathrm{~nm}$ with a side mode suppression of $\approx 26 \mathrm{~dB}$. The emitted light has been collected using collimating lensed fiber with a collection efficiency of $\approx 50 \%$. To implement the delayed feedback, the collected light beam is split into two parts using a $50 \%$ optical coupler: one part is directly read out by a photodiode with $1 \mathrm{GHz}$-bandwidth while the second part is connected to port number 2 of an optical circular. The circulator couples light from port 2 to port 3 and from port 3 to port 1 with minimal power loss. The signal at port number 3 is delayed by a single mode optical fiber of length $\approx 126 \mathrm{~m}$ (i.e., time-delay of $\sim 630 \mathrm{~ns}$ ). The light beam at the fiber output is split into two paths. One path passes through a $10 \mathrm{GHz} \mathrm{LiNbO}_{3}$ phase modulator (PM) (half-wave voltage $V_{\pi}=5 \mathrm{~V}$, maximum input power $28 \mathrm{dBm})$. The PM output signal is combined with the light beam of the second path using a $2 \times 2$ optical coupler implementing an intensity modulation, of the light beam. One output port of this coupler is connected to port number 1 of the circulator while the second output port is connected to an optical spectrum analyzer. So, taking into account the loses about $10 \%$ of the collected power is re-injected into the laser.

The original data to be processed is first convoluted with a random mask which has 4 discrete values $(-1,-0.25,0.25,1)$ generated randomly with equal probability at the time interval of $\theta=24 \mathrm{~ns}$. The resulting signal is uploaded in a two channel arbitrary waveform generator (AWG) (Tektronix AFG3102C, dual channel, $1 \mathrm{GS} / \mathrm{s}, 100 \mathrm{MHz}$ ) from where it is injected into the reservoir via the PM radio-frequency electrode. The amplitude of the injected signal is rescaled such as to obtain an 

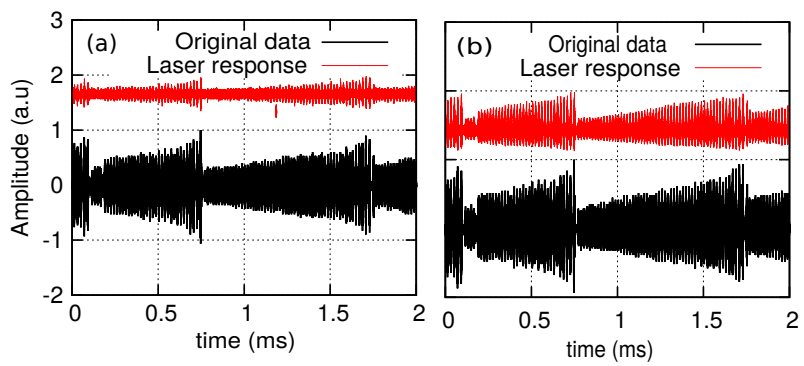

Fig. 3. Original data (black) and the corresponding experimental recorded signal of the laser response read by the photodiode (color) for the diode pump current (a) $I=120 \mathrm{~mA}$ and (b) $I=220 \mathrm{~mA}$. The amplitude of the input data and the laser response have been rescaled with its maximum value in each case. The laser response signal has been shifted for clarity.

optimal contrast of the modulation at the laser output. In our case, the peak-to-peak voltage from the AWG was $10 \mathrm{~V}$. The data is recorded using a digital oscilloscope (LeCroy $200 \mathrm{MHz}$, $2.5 \mathrm{GS} / \mathrm{s})$. The AWG is perfectly synchronized with the oscilloscope so that the original data and the laser response to this data can be simultaneously recorded. We experimentally use 1000 steps for training and 250 steps for testing. The samples are injected at the time interval $T_{d}=2.4 \mu \mathrm{s}$ which is 3.8 times larger than the time-delay, i.e $T=630$ ns. This corresponds to a reservoir with $N=T_{d} / \theta=100$ virtual nodes. As such, only a fraction of the virtual nodes (i.e., $\sim N / 3.8$ ) are connected through the feedback to nodes in a previous state (i.e. nodes that receive an input corresponding to a previous time).

Figure 3 shows the temporal profiles of the original data (black) and the laser response at the photodiode output (red) for two values of the diode-pump currents with one close the threshold $(I=120 \mathrm{~mA})$ and another far away from the threshold $(I=220 \mathrm{~mA})$. For $I=120 \mathrm{~mA}$, the micro laser emits a low power and therefore the fraction of the signal (i.e., about $10 \%$ of the collected power) to be re-injected into the laser is small. In this case, the dynamical response of the laser to input signal may also be limited since the feedback light beam is the one carrying the input data. For $I=220 \mathrm{~mA}$, the beam fraction driven the data is larger. The laser response is quite similar to the input signal. These laser output signals are used to optimize the readout weights. The prediction error rates NMSE obtained for the two cases were $\approx 0.4$ and $\approx 0.07$ for $I=120 \mathrm{~mA}$ and $I=220 \mathrm{~mA}$ respectively. To demonstrate that the nonlinearity of the laser is necessary for this computation, we have also optimized the weights directly from the AWG's output signals. We obtained the prediction error rates NMSE of $\approx 0.7$. This significantly worse performance evidences that eventual nonlinearity caused by the out-of-bandwidth operation of the AWG does not already allow for reservoir computing.

To identify the most suitable parameter regimes for which the system can successfully predict a chaotic input signal one-time step ahead in the future, we show in Fig. 4 the system performance expressed by the NMSE as a function of the diode-pump rate $\mu=\left(I-I_{s p}\right) /\left(I_{s}-I_{s p}\right)$. For each value of $\mu$, we run the experiments 6 different times and record for each run a time trace of $5 \mathrm{~ms}$ at the output of the photodiode. The sampling rate at the oscilloscope is $2 \mathrm{~ns}$. The shown values of the NMSE are the mean values over the runs. For low pump rates $\mu$, large values of NMSE are obtained meaning worse performance. This degradation is due to noise which is dominant for small laser output powers (the signal-to-noise ratio (SNR) is small in this case).
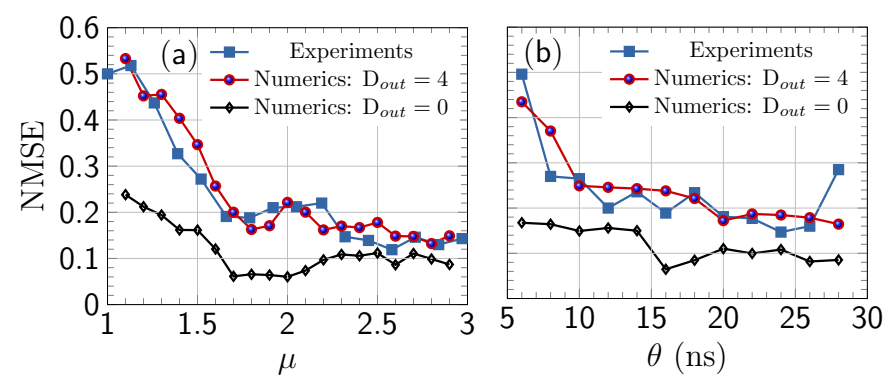

Fig. 4. Prediction errors NMSE as a function of (a) the pump rate $\mu$ for virtual node's separation $\theta=24 \mathrm{~ns}$; (b) $\theta$ for $\mu=2.5$. The time interval resolution of the time-delay is $\theta$.

This noise is mainly readout noise from photodiode. For large values of $\mu$ (i.e., large laser output signals), the SNR is large and the influence of readout noise can be minimized. For such cases, we obtain a lowest NMSE of $0.12 \pm 0.04$ at $\mu \approx 2.58$ (note that for clarity, the error bars are not plotted) while a lowest NMSE of 0.108 was obtained in [7] for the same task. The performance of the two systems is therefore similar although we consider a time delay comparable to the laser's relaxation oscillation time, $\tau_{R O}$, [note that $\tau_{R O} \approx\left(\gamma \gamma_{e}(\mu-1)\right)^{-1 / 2} \approx 750$ ns at $\mu \approx 2.5$ ].

For real-world applications, the delay length would be fixed during the manufacturing phase of the device while the length of the mask could be independently varied by changing, for example, the temporal node's separation. To investigate this fact, we show in Fig. 4(b) the NMSE values for different values of $\theta$ when $N$ and $\mu$ are kept fixed to 100 and 2.5 respectively. The optimal value lies around $\theta=24$ ns. These are therefore the values of $\theta$ for which the optimal coupling between the virtual nodes is obtained.

For further insights, we perform numerical simulations using the model of diode-pumped Erbium microchip laser subject to optical delayed feedback introduced in $[20,21]$. The relavant variables of the model are the slow-varying complex envelope of the electric field $E_{m}(t)$ associated to the longitudinal mode $m$ (with $m=1,2$ ) and its corresponding carrier number $N_{m}(t)$ :

$$
\begin{aligned}
\dot{E}_{1,2} & =\frac{1}{2}\left(B g_{1,2} N_{1,2}-\gamma\right) E_{1,2}+\frac{i \alpha}{2} B N_{1,2} \\
& +\frac{\eta}{2}\left[1+e^{i\left(s(t)-\omega_{1,2} \delta T\right)}\right] E_{1,2}(t-T) e^{i \omega_{1,2} T}+\xi_{1,2}(t), \\
\dot{N}_{1,2} & =\gamma_{e}\left(N_{p}-N_{1,2}\right)+g B N_{1,2}\left(\left|E_{1,2}\right|^{2}+\varrho\left|E_{2,1}\right|^{2}\right)+\zeta_{1,2}(t),
\end{aligned}
$$

where the parameters are the Einstein coefficient: $B$, the decay rate of the population inversion: $\gamma_{e}$, the pumping rate: $\gamma_{e} N_{p}$, the laser cavity decay rate: $\gamma$, the linewidth enhancement factor: $\alpha$, the feedback rate: $\eta$, the time delay: $T$, the cross-saturation parameter: $\varrho$, the solitary laser frequency for mode $m: \omega_{m} . S(t)$ is the signal which results from convolution between the original data to be processed and the mask. The noise is modeled as the Langevin forces $\xi_{1,2}(t)$ and $\zeta_{1,2}(t)$ which describe the quantum fluctuations of the laser population and the radiation field. These forces are defined as having a zero mean value and whitenoise-type correlation functions: $\left\langle\xi_{i}(t) \xi_{j}^{*}\left(t^{\prime}\right)\right\rangle=D \delta_{i j}\left(t-t^{\prime}\right)$ and $\left\langle\zeta_{i}(t) \zeta_{j}^{*}\left(t^{\prime}\right)\right\rangle=D \gamma \gamma_{e} \mu \delta_{i j}\left(t-t^{\prime}\right) / B$ for $i, j=1,2$ where $D$ is the spontaneous emission factor.

From experiments, we have retrieved the parameters $\gamma=$ $53.66 \mu \mathrm{s}^{-1}, \gamma_{e}=0.217 \mu \mathrm{s}^{-1}, \varrho=0.43$. We also consider other parameters from [20, 21]: $B=122.1, \alpha=1, \eta=0.3 \mu \mathrm{s}^{-1}$, $g=0.95, N_{p}=\mu \gamma / B, \omega_{m} T=0, \omega_{m} \delta T=-0.9 \pi$ and $D=10^{-4}$. 

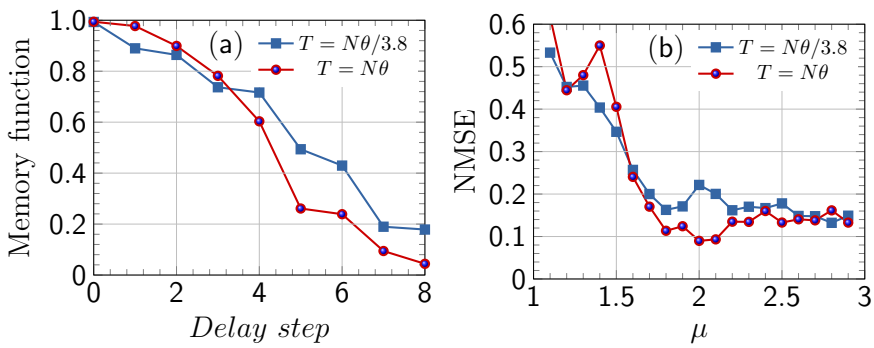

Fig. 5. Numerical results: (a) Memory function for two values of time-delay: For $T=N \theta$, all the $N$ virtual nodes are connected to nodes in the previous state. For $T=N \theta / 3.8$, only $N / 3.8$ virtual nodes are connected to nodes in the previous state. (b) Corresponding NMSE as a function of the pump rate $\mu$. We consider $\theta=24$ ns and $\mu=2.5$. The time interval resolution of the time-delay is $\theta$.

We numerically use 3000 steps in the data set for training and 1000 other steps for testing. For each value of $\mu$, we run the simulations 10 different times and record for each run the laser output as $|E(t)|^{2}=\left|E_{1}(t)+E_{2}(t)\right|^{2}$. Additional Gaussian white noise is added to $|E(t)|^{2}$ to model the readout noise from the photodiode. Thus, the weights are optimized from $|E(t)|^{2}+D_{\text {out }} \xi_{\text {out }}(t)$ instead of $|E(t)|^{2}$, where $D_{\text {out }}$ is the noise amplitude while $\xi_{\text {out }}(t)$ is a Gaussian white noise with zero mean and correlation $\left\langle\xi_{\text {out }}(t) \xi_{\text {out }}^{*}\left(t^{\prime}\right)\right\rangle=\delta\left(t-t^{\prime}\right)$. $D_{\text {out }}=0$ refers to noiseless photodetectors. We choose $D_{\text {out }}=4$ as it is the value for which numerical and experimental SNRs calculated as $\mathrm{SNR}=10 \log _{10}\left(\left\langle|E(t)|^{2}\right\rangle /\left\langle D_{\text {out }}\right\rangle\right)$ are similar. The SNR is $\approx 10 \mathrm{~dB}$ at $\mu=1.2$ and $\approx 26 \mathrm{~dB}$ at $\mu=2.5$. The value of $D_{\text {out }}$ is kept fixed for the rest of the Letter.

The numerical obtained values of the NMSE as a function of the pump rates are also shown in Fig. 4 in comparison with the experimental ones. Again, the error bars are not plotted. In both cases, an excellent agreement is found between experimental and numerical results. In particular, it is confirmed that, because of noise, the system performance strongly degrades for small values of $\mu$ [see Fig. 4(a)] and also for high processing speeds [see Fig. 4(b)]. We obtain, for example, an NMSE $\approx 0.43$ for $\theta=6 \mathrm{~ns}$ although all the $N$ virtual nodes are connected through the feedback to nodes in the previous state for this $\theta$. Note that for fixed $N$, the processing speed increases with the decrease of $\theta$. By comparing the NMSE values obtained from a system with $(\bullet)$ and without readout noise $(\diamond)$, it turns out that the degradation of the NMSE for low values of $\mu$ is mainly due to the readout noise. Through numerical simulations, we have also found that the calculation of the weights from $\left|E_{1}(t)\right|^{2}$ or $\left|E_{2}(t)\right|^{2}$ (i.e., considering only one mode) yields similar results as from $\left|E_{1}(t)+E_{2}(t)\right|^{2}$. This is not surprising since the mode spacing is large such that the heterodyne signal is outside the detection bandwidth of the system. In such a case, the choice of either mono- or bi-mode laser is not of importance.

The memory capacity of the system may decrease when only a fraction of the virtual nodes is connected through the feedback to the previous input states. To investigate this fact, we compare in Fig. 5 (a) the simulated results of the memory function (calculated from uniformly distributed random signal drawn in the interval $[-0.5 ; 0.5])$ when $N / 3.8$ virtual nodes $(\boldsymbol{\square})$ and when all the $N$ virtual nodes $(\bullet)$ are connected to nodes in a previous state considering $\theta=24$ ns and $\mu=2.5$. Effectively, it is seen that the memory slightly decreases for $N / 3.8$ connected to nodes in a previous state. The fading slope of the memory is also slow in this case. But, the calculation of the NMSE shows that these changes in the memory do not significantly degrade the system performance for one-step ahead prediction [Fig. 5 (b)]. However, the low memory in Fig. 5 (a) suggests that this system may not be suitable for some tasks requiring large memory capacity.

In conclusion, we have experimentally and numerically shown that diode-pumped Erbium doped microchip lasers subject to optical feedback can be used to implement RC systems for prediction tasks. Using Santa-Fe time series as benchmark, we found a best prediction error similar to that obtained with a similar system (but with a long time-delay [7]) although the use of a short time-delay. Our results also have shown that even multi-mode lasers with large mode spacing can be used.

\section{FUNDING}

This work has been supported by the F.N.R.S. (Belgium). R.M.N acknowledges the support CNRS during his stay at LIPhy. This work also benefited from the support of the Belgium Science Policy Office under Grant No IAP-7/35 "photonics@be".

\section{REFERENCES}

1. W. Maass, T. Natschläger, H. Markram, Neural Comput. 14, 2531 (2002).

2. H. Jaeger and H. Haas, Science 304, 78 (2004).

3. K. Vandoorne, P. Mechet, T. V. Vaerenbergh, M. Fiers, G. Morthier, D. Verstraeten, B. Schrauwen, J. Dambre, and P. Bienstman, Nat. Commun. 5, 4541 (2014).

4. L. Appeltant, M. C. Soriano, G. Van der Sande, J. Danckaert, S. Massar, J. Dambre, B. Schrauwen, C. R. Mirasso, and I. Fischer, Nat. Commun. 2, 468 (2011).

5. L. Larger, M. C. Soriano, D. Brunner, L. Appeltant, J. M. Gutierrez, L. Pesquera, C. R. Mirasso, and I. Fischer, Opt. Express 20, 3241 (2012).

6. Y. Paquot, F. Duport, A. Smerieri, J. Dambre, B. Schrauwen, M. Haelterman, and S. Massar, Sci. Rep. 2, 287 (2012).

7. D. Brunner, M. C. Soriano, C. R. Mirasso, and I. Fischer, Nature Commun. 4, 1364 (2013).

8. M. C. Soriano, S. Ortín, D. Brunner, L. Larger, C. R. Mirasso, I. Fischer, and L. Pesquera, Opt. Express 21, 12 (2013).

9. F. Duport, B. Schneider, A. Smerieri, M. Haelterman, and Serge Massar, Optics Express 20, 22783 (2012).

10. A. Dejonckheere, F. Duport, A. Smerieri, L. Fang, J.-L. Oudar, M. Haelterman and S. Massar, Opt. Express 22, 10868 (2014).

11. Q. Vinckier, F. Duport, A. Smerieri, K. Vandoorne, P. Bienstman, M. Haelterman, and S. Massar, Optica 2, 438 (2015).

12. K. Hicke, M. A. Escalona-Moran, D. Brunner, M. C. Soriano, I. Fischer, and C. R. Mirasso, J. Sel. Top. Quantum Electron. 19, 1501610 (2013).

13. R. M. Nguimdo, G. Verschaffelt, J. Danckaert, and G. Van der Sande, Opt. Express 22, 8672 (2014).

14. J. Nakayama, K. Kanno, and A. Uchida, Opt. Express 24, 8679 (2016).

15. R. M. Nguimdo, G. Verschaffelt, J. Danckaert, and G. Van der Sande, Opt. Express 24, 1238 (2016).

16. R. M. Nguimdo, G. Verschaffelt, J. Danckaert, and G. Van der Sande, IEEE Trans. Neural Netw. Learn. Syst. 26, 3301 (2015).

17. P. Antonik, F. Duport, A. Smerieri, M. Hermans, M. Haelterman, S. Massar, "Online training of an opto-electronic reservoir computer," Neural Netw. Processing, 22nd International Conference, ICONIP 2015 Istanbul, Turkey, November 9-12, 2015 Proceedings, Part II

18. A. S. Weigend and N. A. Gershenfeld, "Time series prediction: Forecasting the future and understanding the past," ftp://ftp.santafe.edu/pub/TimeSeries/Competition (1993).

19. P. Thony, E. Molva, OSA TOPS on Advanced Solid-State Lasers, 1996, Vol. I , Stephen A . Payne and Clryord Pollock (eds.)

20. E. Lacot, O. Jacquin, O. Hugon, and H. Guillet de Chatellus, Appl. Optics 54, 9763 (2015).

21. C. Szwaj, E. Lacot, and O. Jacquin, Phys. Rev. A 70, 033809 (2004). 


\section{REFERENCES}

1. W. Maass, T. Natschläger, H. Markram, "Real-time computing without stable states: a new framework for neural computation based on perturbations," Neural Comput. 14, 2531 (2002).

2. H. Jaeger and H. Haas, "Harnessing nonlinearity: predicting chaotic systems and saving energy in wireless communication," Science 304, 78 (2004).

3. K. Vandoorne, P. Mechet, T. V. Vaerenbergh, M. Fiers, G. Morthier, D. Verstraeten, B. Schrauwen, J. Dambre, and P. Bienstman, "Experimental demonstration of reservoir computing on a silicon photonics chip," Nat. Commun. 5, 4541 (2014).

4. L. Appeltant, M. C. Soriano, G. Van der Sande, J. Danckaert, S. Massar, J. Dambre, B. Schrauwen, C. R. Mirasso, and I. Fischer, "Information processing using a single dynamical node as complex system," Nat. Commun. 2, 468 (2011).

5. L. Larger, M. C. Soriano, D. Brunner, L. Appeltant, J. M. Gutierrez, L. Pesquera, C. R. Mirasso, and I. Fischer, "Photonic information processing beyond Turing: an optoelectronic implementation of reservoir computing," Opt. Express 20, 3241-3249 (2012).

6. Y. Paquot, F. Duport, A. Smerieri, J. Dambre, B. Schrauwen, M. Haelterman, and S. Massar, "Optoelectronic reservoir computing," Sci. Rep. 2, 287 (2012).

7. D. Brunner, M. C. Soriano, C. R. Mirasso, and I. Fischer, "Parallel photonic information processing at gigabyte per second data rates using transient states," Nature Commun. 4, 1364 (2013).

8. M. C. Soriano, S. Ortín, D. Brunner, L. Larger, C. R. Mirasso, I. Fischer, and L. Pesquera, "Optoelectronic reservoir computing: Tackling noiseinduced performance degradation," Opt. Express 21, 12-20 (2013).

9. F. Duport, B. Schneider, A. Smerieri, M. Haelterman, and Serge Massar, "All Optical Reservoir Computing," Optics Express 20, 22783 (2012).

10. A. Dejonckheere, F. Duport, A. Smerieri, L. Fang, J.-L. Oudar, M. Haelterman and S. Massar, "All-optical reservoir computer based on saturation of absorption," Opt. Express 22, 10868 (2014).

11. Q. Vinckier, F. Duport, A. Smerieri, K. Vandoorne, P. Bienstman, M. Haelterman, and S. Massar, "High-performance photonic reservoir computer based on a coherently driven passive cavity," Optica 2, 438446 (2015).

12. K. Hicke, M. A. Escalona-Moran, D. Brunner, M. C. Soriano, I. Fischer, and C. R. Mirasso, "Information processing using transient dynamics of semiconductor lasers subject to delayed feedback," J. Sel. Top. Quantum Electron. 19, 1501610 (2013).

13. R. M. Nguimdo, G. Verschaffelt, J. Danckaert, and G. Van der Sande, "Fast photonic information processing using semiconductor lasers with delayed optical feedback: role of phase dynamics," Opt. Express 22, 8672-8686 (2014).

14. J. Nakayama, K. Kanno, and A. Uchida, "Laser dynamical reservoir computing with consistency: an approach of a chaos mask signal ," Opt. Express 24, 8679 -8692 (2016).

15. R. M. Nguimdo, G. Verschaffelt, J. Danckaert, and G. Van der Sande, "Reducing the phase sensitivity of laser-based optical reservoir computing systems," Opt. Express 24, 1238-1252 (2016).

16. R. M. Nguimdo, G. Verschaffelt, J. Danckaert, and G. Van der Sande, "Simultaneous computation of two independent tasks using reservoir computing based on a single photonic nonlinear node with optical feedback," IEEE Trans. Neural Netw. Learn. Syst. 26, 3301-3307 (2015).

17. P. Antonik, F. Duport, A. Smerieri, M. Hermans, M. Haelterman, S. Massar, "Online training of an opto-electronic reservoir computer," Neural Netw. Processing, 22nd International Conference, ICONIP 2015 Istanbul, Turkey, November 9-12, 2015 Proceedings, Part II

18. A. S. Weigend and N. A. Gershenfeld, "Time series prediction: Forecasting the future and understanding the past," $\mathrm{ftp}: / /$ ftp.santafe.edu/pub/Time-Series/Competition (1993).

19. P. Thony, E. Molva, " $1.55 \mu \mathrm{m}$-wavelength CW microchip lasers," OSA TOPS on Advanced Solid-State Lasers, 1996, Vol. I, Stephen A . Payne and Clryord Pollock (eds.)

20. E. Lacot, O. Jacquin, O. Hugon, and H. Guillet de Chatellus, "Control of the differential interference contrast in reinjected bimode laser," Appl. Optics 54, 9763-9771 (2015).
21. C. Szwaj, E. Lacot, and O. Jacquin, "Large linewidth-enhancement factor in a microchip laser," Phys. Rev. A 70, 033809 (2004). 